\title{
Multi-organisational approach to safety training: the case of a Finnish Safety Training Park
}

Studies across trades suggest that occupational accident and injury rates and costs are higher than average in the construction industry. There is, a need for innovative and cost-effective occupational safety approaches. The Finnish construction industry and its key stakeholders have introduced a new safety training concept, called the Safety Training Park (STP). The STP consists of fullscale training points that use new training methods to stimulate individual safe behaviour and safety climate changes within work groups and companies. The Safety Training Park in Northern Finland (STPNF) was designed, constructed, and financed through a collaborative process that included over 80 organizations. A realistic evaluation approach was applied to document the effectiveness of STPNF training from individual and organizational perspectives. Results indicate that the dialogical STP approach with several information sharing channels fits the target groups. Evidence regarding effectiveness at the organizational level is also discussed. Cost-benefit calculations show that the multi-organizational coalition model enables efficient occupational safety and health training with reasonable costs.

Keywords: collaboration, construction education, effectiveness, information sharing, occupational health and safety, realistic evaluation, stakeholder, training

\section{Introduction}

The construction industry is an important global contributor to both employment and the economy (Lehtola et al. 2008). The industry typically accounts for 7-12\% of a country's employment and Gross Domestic Product but also accounts for 20-30\% of serious work-related injuries. Additionally, it has at least an equivalent share of occupational illnesses and musculoskeletal disorders (Ringen et al. 2010). In general, analyses from different countries show a downward trend in occupational accidents and injuries, but no significant changes are seen within the construction industry (Lander et al. 2016). Besides human suffering, occupational accidents and injuries cause financial 
losses to the employee, the employer, and society. Costs are diverse, including direct cost elements like sickness payments, increased insurance premiums, administrative and legal fees, damaged equipment, environmental hazards, production losses and indirect costs like disturbances, decreased human productivity and a lowered company reputation (Rikhardsson 2004, Manuele 2011).

Construction sites differ from normal work environments, as they evolve continuously (Andersen et al. 2018). In addition, construction sites are multiorganizational workplaces where various employers and their employees act simultaneously (Swuste et al. 2012, Andersen et al. 2018). The complex nature of construction sites induces specific challenges to occupational safety and health (OSH) practices and processes. It is obvious that new approaches are needed to reduce the number of accidents and injuries in the construction sector.

Different kinds of training are often used in developing individuals' OSH skills and knowledge (Burke et al. 2006, Robson et al. 2012). The Finnish construction industry and its key stakeholders have developed a novel concept: the Safety Training Park (STP). The Rudus construction company created the first STP in southern Finland in 2009. Another STP was built in northern Finland (STPNF) in 2014 (Reiman et al. 2019a). Both of these STPs demonstrate real work environments (i.e., training points with safety dilemmas) and use innovative OSH training methods to stimulate changes in individual's safety behaviour and to improve the safety climate at work group, work site and company levels. Safety climate is a complex concept that can be discussed at work group, construction site and company levels. It is based on the shared conceptions of the relative prioritizing of safety compared to other competing priorities (Zohar 2010). Thus, the safety climate relates to the procedures, practices, and types of behaviours that are rewarded and supported concerning a specific strategic focus (e.g., Zohar 2010). It 
has been documented that safety climate is a robust indicator or predictor of OSH outcomes across industries and countries, including the construction sector (Kines et al. 2010, Zohar 2010).

To respond to the recent academic debate about the opportunities and challenges to develop the safety climate in construction (Andersen 2018) and to identify efficient OSH training approaches (Ricci et al. 2016, Hofmann et al. 2017), scientific studies should thoroughly examine the effectiveness of training at different levels and from different perspectives. This study aims to contribute to these discussions. This study uses a realistic evaluation model to evaluate the effectiveness of the STPNF training from trainee and company perspectives, respectively. The analysis is structured around two research questions:

(1) How do participants of different ages, genders, and occupational groups experience the STPNF training?

(2) Are there any signs of company-level effectiveness based on accident statistics?

\section{Review of OSH Training}

As construction sites are often multi-organizational environments, the safety climate may evolve not only from the companies' own practices and processes but also work group and site-specifically (Andersen et al. 2018). Additionally, employees play a major role in determining their own safety through their implementation and interpretation of safety practices and processes (Sacks et al. 2013). Thus, a key objective of OSH training is to affect personal behaviour by improving awareness of OSH hazards, expanding knowledge of the causes of illness, injuries and accidents, and promoting the implementation of effective preventive measures (e.g., Komaki et al. 1980, Arnold et al. 2005, Burke et al. 2006). In this context, relevant characteristics of 
trainees in the construction sector should be acknowledged. Employees tend to be lesseducated (Wilkins 2011), male (Zou and Sunindijo 2015), and risk takers (Phua 2017). Construction sites are often multinational, and language barriers for employees from different countries and cultures might exist (Loosemore and Lee 2002, Demirkesen and Arditi 2015).

The range of OSH training methods varies from passive information-based techniques, such as lectures and courses, videos, cartoons, and written materials to learner-centred and performance-based techniques (Burke et al. 2006, Ulubeyli et al. 2015). Learner-centred active techniques in the construction sector include traditional hands-on practices and modern approaches, such as virtual reality simulation, computerassisted learning, e-learning, remote learning, and online learning (Ho and Dzeng 2010, Sacks et al. 2013, Demirkesen and Arditi 2015, Zhao and Lucas 2015).

In general, researchers are in favour of techniques that stimulate trainee participation being more effective than passive techniques (Burke et al. 2006). Furthermore, methods that are tailored to trainee demographics and provide opportunities for dialogue and feedback seem to be the most efficient in the OSH training context (Komaki et al. 1980, Dong et al. 2004, Burke et al. 2006, Burke et al. 2011). However, the effectiveness of OSH training is still unknown (Hale 1984, Robson et al. 2012, Ricci et al. 2016).

Learning is an important dimension of training (Demirkesen and Arditi 2015). In addition, the transfer of learning must be considered. Even though employees may acquire new knowledge and skills through training, they might not apply or use these when they return to work (Arnold et al. 2005). The safety climate at both the organizational level and at the team level contributes to the transfer of learning to the work sites (Zohar 2010). In practice, the safety climate is closely related to 
organizational factors such as the actual support and opportunities to utilize new skills and knowledge at the actual work environment where the trainee is working (Arnold et al. 2005).

Based on Kirkpatrick’s (1959) hierarchical categorisations on evaluating training the influences may be realised at four levels. Firstly, training may induce instant reactions (e.g., satisfaction with the training). Secondly, training may lead to learning and subsequent changes in attitudes. Thirdly, training may induce behaviour changes in practice (e.g., transfer of learning in practice). Fourthly, successful training will eventually have an impact on the organisation's key performance indicators (KPIs) (Kirkpatrick 1959).

\section{Safety Training Park concept}

This study focuses on STPNF. The STPNF was designed, constructed, and financed through multi-stakeholder cooperation of over 80 organizations (referred to as the STPNF coalition). The organizations belonging to the STPNF coalition cover a wide range of different actors, including major construction companies, several small and medium-sized construction companies, construction product companies and service providers. Other actors in the coalition include local schools, universities and other educational institutions as well as companies from other industries, municipalities, insurance companies, and governmental parties (see Reiman et al. 2019b). The STPNF design and construction process was based on participatory ergonomics (BurgessLimerick 2018) at different levels. Members of the coalition jointly determined the overall structure of the STPNF, the types of OSH issues that should be covered, and how the training would be delivered.

STPNF is a physical learning environment, covering 1.6 hectares. The area is divided into training points (see Table 1) where construction-specific OSH issues are 
demonstrated. Most training points include scenarios that demonstrate good or dangerous work practices. Dummies, signs, and posters are commonly used as additional information sharing channels to enliven the demonstrations (see Table 1). Figure 1 shows a training point that demonstrates transits and transportations at construction sites. In Figure 1, a dummy and a crashed car show the dangers related to potential shadow areas from the cab of the vehicle. Another dummy is used to initiate discussion on good practices for how to approach a vehicle at the site. In addition, trainees can participate in kinesthetic learning that teaches good practices for ascending and descending a cab.

[Figure 1 near here]

[Table 1 about here]

STP training is based on dialogic learning and sharing of experiences (e.g., Isaacs 1993). Using the dialogic learning approach, trainers encourage trainees to identify and discuss their observations at the training points. The trainers then focus the discussion towards the trainee's own experiences on the subject. This approach is assumed to improve personal and work group level risk awareness and hazard and discomfort identification skills.

Currently, over 200 in-house trainers have been certified to provide training at the STPNF. The trainer group is heterogeneous. However, most of the trainers are either OSH professionals or middle managers coming from the coalition organizations. The STPNF coalition has provided common instruction for STPNF training to happen within a four-hour training session covering all training points. However, the trainers are also strongly encouraged to tailor training to meet the needs of the trainees. 
Approximately 5,000 trainees visit STPNF annually. Roughly half of the trainees work for companies, and half are students from different levels.

\section{Methods and materials}

To provide a comprehensive evaluation of the effects of STPNF training, a combination of qualitative and quantitative methods and material was used. Methodology in this study was based on the realistic evaluation framework.

\section{Realistic evaluation framework}

Effectiveness evaluation in the context of this study requires identifying and understanding the effects at individual and company levels. Traditional evaluation research views randomized controlled trials (RCTs) as the golden standard and mainly focuses on whether the expected results appear or not. Previous studies highlighted the limitations of this type of evaluation, especially in dynamic organizational interventions (Baker et al. 2001, Sanson-Fisher et al. 2007, Nielsen and Randall 2013). Recently, realistic evaluation has received attention in the $\mathrm{OSH}$ literature as a new way to design and evaluate OSH interventions while still adhering to the basic principles of the Cochrane review criteria (Pedersen et al. 2012, Yassi et al. 2013, Kvorning et al. 2015). Realistic evaluation asks what works for whom and under what circumstances? (Pedersen et al. 2012). Additionally, realistic evaluation aims to open the "black box" of OSH interventions by including information regarding the implementation, context, and mechanisms, and their influence on intervention outcomes. Qualitative or quantitative data can be used to develop valid measures of context, mechanisms, and intervention effects (Pedersen et al. 2012). Mechanisms are defined as the "drivers of change," such as relevant personal characteristics of the key actors (e.g., trainers or trainees) in the intervention (Pedersen et al. 2012). Realistic evaluation distinguishes between theory 
failure and implementation failure. Implementation failure occurs when the intervention is unsuccessful due to a lack of participation (i.e., the intervention works, but the target audience does not engage in it). Theory failure occurs when the intervention is implemented correctly but does not have the expected outcome (i.e., the target audience participates, but the intervention does not work), which points at failure in the underlying program theory (Pedersen et al. 2012).

In this study, the program theory was based on the theories of learning (Kirkpatrick 1959) and safety climate (Zohar and Luria 2005). Effectiveness was measured as a two-dimensional phenomenon at the individual and company levels, respectively. Effectiveness at an individual level was measured as self-perceived reactions and following intentions to changing behaviour. In addition aspects that have facilitated or hindered this learning process and further transfer of learning were identified. A questionnaire aimed for all STPNF trainees and interviews for selected trainee groups were used as a data collection method for this purpose. As a measure of safety climate development company-specific performance indicators concerning annual accident statistics were collected. Accident statistics were compared to statistics from the Finnish construction industry and the analysis was supplemented by a scenario calculation of the economic costs and benefits of the STPNF training.

To reduce bias in selecting companies from which company-specific data was collected (Pedersen et al. 2012), the researchers only knew that the companies had utilized the STPNF; they did not know their OSH performance status. Many different types of organizations were utilizing the STPNF (Table 2); thus small, medium-sized, and large construction companies with different ownership arrangements were chosen. Also, construction service providers of different sizes and backgrounds were included. Data collection was limited by companies' willingness to share information. 
[table 2 near here]

\section{Questionnaire}

A questionnaire containing both quantitative and qualitative questions was given to STPNF trainees during 2014-2015. The questionnaire provided useful information regarding the implementation of the training and trainees self-perceived satisfaction of each training point and the STPNF. This data, combined with background information regarding position, age, and gender, enabled identifying differences and similarities in the trainee groups and thereby come closer to answering what works for whom?

Questionnaires were filled out immediately after the training, but they were voluntary, and distribution depended on the trainers' willingness to collect feedback. The questionnaire form included three sections: 1) background information, 2) evaluation for each training point $(\mathrm{n}=21)$ on a scale from 1 (improvements needed) to 5 (good training point design), and 3) feedback regarding training experiences. Additionally, the respondents were asked whether they would apply any new knowledge or skills they learned through the training.

The data consists of 1,167 questionnaire forms with a response rate of around 17\% (see Table 3).

[Table 3 near here]

Average values and a Kruskal-Wallis test with IBM SPSS 24 were used to study the differences between the groups. The level of statistical significance was defined as $\mathrm{p}<$ 0.05 . 


\section{Interviews}

Focus group interviews (Cooper and Baber 2005) were held to get more detailed information about STPNF training effectiveness. Trainees from companies A, B, C, and D were interviewed (see 4). The training were conducted by certified in-house trainers who followed the four-hour training course. Focus group discussions aimed to identify aspects that facilitated or hindered learning and learning transfer. To identify whether perceptions varied over time, each focus group was interviewed twice; about one month and one year after the training. In addition, personal interviews were conducted with representatives of top management.

[Table 4 near here]

Interviewees were selected in cooperation with the target companies' human resources and OSH specialists. Selections were based on each company's training schedule. Employee recruitment for company D was site-specific. Thus, the same interview group was reached only once. In addition, changes in top management prevented personal interviews. The interviews lasted from 48 to 92 minutes, and they were conducted in a closed room at the company office or construction sites. Interviews were recorded and transcribed. In the interview analysis phase, two researchers independently identified and categorized respondents' views on factors that supported or hindered learning. To augment the analyses, direct quotations from the interviews are used in this article (translated from Finnish to English by the first author).

\section{Company-specific performance indicators}

Objective organizational level performance indicators are needed to support claims regarding training effectiveness (Kirkpatrick 1959). In this study, accident statistics 
were used as organizational level indicators, and accident statistics from the Finnish construction industry (Confederation of Finnish Construction Industries RT, 2018) were used as a reference. Information about company-specific accident statistics between 2011 and 2016 was requested via a web page inquiry in winter 2017 from altogether 35 construction companies or their service providers belonging to the STPNF coalition. Altogether seven companies replied by providing data covering years 2011 to 2016; thus, the response rate was $20.0 \%$. The accident statistics required included annual working hours and the number of all occupational accidents that had led to more than one day absence from work. The annual accident frequency (accidents per million working hours) was calculated by dividing the total amount of accidents by the total amount of working hours and multiplying that by a million.

Annual changes in accident frequency were considered as objective signs of changes in the safety climate at the company level. As Hendrick (2008) emphasizes, efforts should be made to express OSH investments in financial terms. At the end of this article, a rough cost-benefit analysis is included to highlight the potential for economic effectiveness of STPs at the company level. Costs include annual membership fees (2,500 euro), training point design and construction expenses (approximation based on the interviews), and personnel costs regarding training. Personnel costs were calculated using an average value of 300 euro for a lost working day in Finland (refer to Seppänen 2009). The benefits were based on estimations of potentially avoided accident costs, assuming that an average occupational accident in the Finnish construction industry results in direct company costs of around 7,000 to 8,000 euro (Reiman et al. 2019c). 


\section{Results}

\section{Trainee satisfaction}

As mentioned, construction workers have diverse OSH backgrounds, perspectives, and experiences. Hence, it is relevant to learn about trainees' experiences and satisfaction with the training. For that purpose, the respondents' satisfaction concerning the training points in the STPNF were analysed from the questionnaire data (see Table 5). The average values for each training point were high, ranging from 3.58 to 4.45 , as measured on a scale from 1-5. Most respondents ( $\mathrm{n}=442)$ said that they would apply new knowledge or skills acquired from the STPNF training in their daily work which indicates potential for long-term changes in the safety climate. Only eight respondents (four students and four employees) said that they do not know or are not going to apply any new knowledge to their work.

[Table 5 near here]

Compared to men, women provided the highest values of satisfaction at all training points, and statistically significant differences were found for eleven training points (highlighted in Table 5). When respondents from construction and industry branches were compared, the construction branch provided lower values of satisfaction for twelve training points. Statistically significant differences were found for six training points. There is no single explanatory factor in these six training points that would exactly discriminate them from others. An overarching aspect connecting these six training points could be that they can be considered containing training aspects that reach outside the working hours. However, this also applies to some other training points. Three out of these six training points, namely Construction work for house technology 
(Training point 4), Property maintenance (9), and Personal protective equipment (PPE) exhibition (17) all cover issues that can be associated with small maintenance and repair issues that are also performed outside working hours. In addition, Transits and transportation at sites (10), Traffic control in road work (13) and Asphalt work (14) can be considered to cover general road safety issues in addition to branch-specific OSH aspects. All of the six training points contain posters and present good OSH solutions; however, there are some minor differences in the utilization of demonstrated work environments and dummies.

An analysis of respondent work status revealed statistically significant differences on satisfaction for training points 3, 5, and 8 (Table 5). Expert respondents showed greater satisfaction toward STP training points as their average values were the highest for 16 out of 21 training points (76.2\%). The group of experts consisted of OSH professionals, who naturally should have a more positive attitude toward OSH and accident prevention. Respondents from the management and student groups were the most critical, providing the lowest average values for satisfaction for 8 and 10 training points, respectively. As an interpretation describing these findings, the critical awareness of training techniques should be acknowledged. Students might be more aware of new techniques available in general and evaluate the training points from that perspective, whereas employees might have more realistic perspectives on the limitations related to actual transfer of learning in practice. Age group analysis revealed statistically significant differences in satisfaction for training points 1, 8, 10, 13 and 15 (Table 5). Middle-aged respondents were the most critical, providing the lowest average values for 12 training points. Young respondents gave seven training points the lowest average values. 
Overall, results show that the respondents are generally very satisfied with the training points. The analysis above suggests that the training has been well implemented and there are no indications of any theory failure. However, there are differences how different target groups gain the training. In summary, it can be stated that women, experts, and respondents over 45 years old, had a more positive view and greater satisfaction of the training points. Men, young and middle-aged respondents, students, and managers were more critical. Following the realistic evaluation approach of linking implementation, results and the work force characteristics in the construction branch, these findings are important when interpreting the effectiveness of the training and its' possible effects on safety climate. However, other measures are needed to achieve more comprehensive understanding about effectiveness at different levels.

\section{Learning and behaviour changes}

Trainee experiences and expectations concerning the training were discussed more indepth in the interviews. Illustrative demonstrations of work environments were considered as aspects that facilitated good learning at individual levels. These aspects were emphasized both one month and one year after the training; thus, they also showed potential for long-term learning. The impact of realistic demonstrations was pointed out in an interview with a regional director: "Everybody has said [to him] that the demonstrations are efficient. They are concrete in showing what can go wrong. For quite many people such horror scenes are a good way to influence." Another regional director expected these demonstrations to change behaviour: "That it should provide a wake-up, like you can't perform certain tasks in that manner or suchlike. It really is possible to have that kind of an accident. In a way, it should stick in one's mind and affect behaviour in such way. That would be a positive way to influence". As an example of behaviour change, an employee expressed how he would apply the good 
practices presented in the training sessions: "As I'm working at heights constantly, it was good to see the variety of different good practices shown there [in the STPNF]. Now it is easier for me to apply that knowledge in practice while working."

Respondents recognized the value of STPs as a forum for in-depth discussions both one month and one year after the training sessions, which shows the positive impact of the dialogic approach on long-term learning. Trainer skills and competence were discussed more in-depth only in the interviews one month after the training. One year later, the focus was changed, so a specific emphasis was not given to the trainers. This may be a sign that the training points themselves and related discussion are the focus of the training. This indicates that the trainers and their individual characteristics and abilities to conduct training are not identified as drivers of change.

Respondents stated that limited opportunities for kinesthetic learning was a barrier that hindered learning. Both one month and one year after the training, interviewees pointed out that hands-on learning opportunities at some training points had enhanced positive experiences at STPNF. This feedback supports the need to develop more engaging OSH training methods, as is also emphasized in the literature.

Realistic evaluation highlights the importance of including expected as well as unexpected results in the evaluation. From that perspective broad level social influencing was expressed as an outcome of the trainings and STPNF itself. The potential for STPs as a broad influencer with expectations on long-term influences was expressed by a regional director: "This [STPNF)] is a reaching out for schools to provide better facilities to teach OSH issues. We think that OSH should definitely be included in curriculums at construction field". These findings indicate that the STPNF training has broader effects than company specific OSH development and accident prevention actions. 


\section{Company level outcomes}

The following analysis focuses company level outcomes and enables discussions regarding improvements in safety climate. Figure 2 shows that the trend in annual accident frequencies was more progressive for the STPNF member companies than for the Finnish construction industry. In 2011, the STPNF member companies involved in this study had 131 lost time injuries leading to at least one day away from work, whereas only 41 accidents occurred in 2016. For STPNF companies, the accident frequency decreased by $63 \%$ during the observation period. However, the reduction was only $42 \%$ for the broader construction industry. This data suggests that companies utilizing STPNF might display better overall OSH performance. Interestingly, accident frequency was lowest among STPNF companies during 2014-2015, which coincides with the opening of the STPNF.

[Figure 2 near here]

Due to contextual differences and underreporting, effectiveness is a complex phenomenon to be measured by objective indicators. This is also true for the companies studied. As expected, manager interviews showed that certain company level costbenefit expectations might exist regarding the utilization of the STPNF. However, the expectations were not explicit, and the complex nature of the calculations was acknowledged.

Potential benefits could be evaluated by different means. In this study, costs and benefits were calculated utilizing national estimations from the construction industry and the STPNF cost data. The costs for utilizing the STPNF vary. STPNF coalition members pay a yearly membership of 2,500 euro. Additionally, training point construction costs, training hours, trainer fees, and travel expenses can be considered as 
major costs in the calculations. We constructed one scenario using a typical Finnish construction company with 50 employees located near the STPNF area. The cost to train all employees annually would be around 11,000 euro, including the annual membership fee (2,500 euro), personnel costs for a half-day long training session $(50 * 150$ euro $=7,500$ euro) and an approximation on the transportation costs and light refreshment during the breaks (1,000 euro). To recover these costs, about 1.5 occupational accidents need to be prevented annually when compared to an average cost of 7,000-8,000 euro for an occupational accident. However, these calculations are rather condensed, and do not account for the long-term impact of training on employee wellbeing and productivity. For example, a trainee might learn a new ergonomic practice for manually handling and lifting materials. This kind of behaviour change focusing on daily actions performed at work sites can prevent accidents at work (and outside working hours), but can also prevent future musculoskeletal disorders in the workplace and at home.

A majority of the companies included in the STPNF coalition provided additional expenses related to designing and constructing the training points. These costs can be considered as occasional investments. When that investment is included in the cost-benefit calculations, the costs rise significantly. Based on manager interviews and their estimations, the design and construction costs were around 10,000-30,000 euro per company. To recover this non-recurring investment, an additional 1.5-4 occupational accidents should be prevented, on average.

\section{Discussion}

OSH challenges in the construction industry are caused by multiple sources ranging from broad societal and organizational factors to individual characteristics (Khosravi et al. 2014). Individual characteristics like immature safety attitudes and motivation, small 
work experience and age have been associated with unsafe behaviours and accidents in construction (Khosravi et al. 2014). However, as Salminen (2004) and Kjestveit (2011) have shown, age is not an unambiguous contributor as both young and old construction employees are prone to accidents. Based on this diversity, not a single OSH training method can appeal to all employees in the construction industry. A need for approaches that combine different training methods is highlighted in the OSH literature.

Burke et al. (2006) conclude that effectiveness studies should focus on situational variables (e.g., safety culture and the transfer of learning) in addition to proximal measures, such as trainee knowledge assessments and satisfaction. If OSH training manage to influence the safety culture and the transfer of learning at the workplace, the training eventually affect behaviours at the job that may, in turn, impact long-term outcomes, such as reduced number of accidents, injuries and illnesses and in the improvements of productivity, thus contributing to the key KPIs of the organisation.

\section{Satisfaction, learning and behaviour changes}

The realistic evaluation framework utilised in this study allows an in-depth discussion of training effectiveness. This study shows that trainees were generally satisfied with the training and the focus group interviews revealed indications of short- and long-term learning. The questionnaire results show that most respondents would make changes to their work. Based on these these findings we can conclude that the first two levels of learning, presented by Kirkpatrick (1959) are fulfilled in the STP trainings.

As far as initiating long-lasting changes in behaviour, as the third level learning category, the question about the transfer of learning in practice arises when changes in broader work group site-specific or company level safety climate is discussed. Verbeek et al. (2011) have shown that effective long-term behaviour changes depend on companies' abilities to support their employees in applying new skills and knowledge. 
Our study did not provide exact insights into barriers that are hindering learning transfer. We see that learning transfer is related to the traditional organization culture in the construction sector. As described by for instance Matinaro and Liu (2017) and Holt (2013) current organizational culture in general in construction hinders development and innovation actions and practices. More detailed knowledge is needed regarding the contextual factors hindering the acceptance of OSH solutions and their utilization at the sites. Only then we can discuss in details STP training contributions to the third level of learning (Kirkpatrick 1959). We see that as STPNF's future research challenge 1 (RC1). When interpreting the results in a learning context, recent discussions on effective learning should be acknowledged. Based on Kirschner (2017), people should not be clustered based on their learning styles. Instead, training should be tailored to the cognitive abilities of the trainee to achieve effective and long-lasting results. STPs follow these recommendations as the training at each training point includes different information sharing channels, and the trainer is authorized to decide based on the trainee group how much time should be spent on each training point. In this context the trainee demographics should be acknowledged. Our material provides insights in that discussion by views on satisfaction concerning the training points by different trainee groups. Our results show that male respondents are slightly more critical of the training points than female respondents. Thus, it is important to consider gender differences when developing STPs. Nielsen et al. (2015) concluded that gender and masculinity are somewhat under-researched within OSH, although the riskiest and dangerous occupations are inherently male-dominated. As the workforce in construction is strongly male-dominated, we suggest that more research is aimed to this area. This is highlighted as STPNF's future research challenge 2 (RC2). 
As STPs can also influence the future workforce, another future research challenge involves developing effective OSH training solutions for young people (RC3). Dong et al. (2004) found that young employees with less working experience are usually more responsive to OSH training and they should be offered a selection of training methods tailored to their age group. As described by for instance Fisk et al. (2009) older adults tend to have more usability constraints and problems when utilizing new technologies than younger adults. The utilization of virtual reality (refer to Sacks $e t$ al. 2013),information and communication technology solutions and gamification in the context of STPs could appeal to young employees and students. However, such solutions might only work for trainees that are familiar with these technologies.

\section{Organizational level outcomes}

Our results show that STPNF training is an effective intervention for improving accident frequency at individual and organizational levels. There is evidence that STPNF companies had managed to decrease their accident frequency in the observation period more progressively than the Finnish construction industry in general. We see this as a reflection of the training effectiveness reaching all the way to the organization level KPIs and accordingly attaining the fourth element of learning by Kirkpatrick (1959). However, as Klotz (2013) pointed out workplace statistics are usually influenced by various other contextual factors. This complicates any interpretations of the exact effects of the training at organisation level. Contextual factors may be realised at various levels.

In addition, the number of companies providing company-specific OSH performance indicators was limited. Further, the sample might be biased as it is possible that only the companies with a good OSH culture responded. However, the national indicators for the construction industry could have been similarly biased as they were 
based on voluntary surveys. In addition, surveys were only sent to companies belonging to the national employer association. Thus, the national sample is somewhat overrepresented by larger companies with better opportunities to improve OSH.

Weak OSH statistics in construction and a higher awareness of the costs of bad OSH have stimulated STP development processes. From the company perspective STPs can be discussed as investments. As emphasized by Hendrick (2003), any OSH development project includes various elements that directly or indirectly hamper estimations of total costs and benefits. This study estimates these costs and benefits to facilitate discussions for detailed cost-benefit calculations of the STPs in the future. Cost-benefit calculations are identified as the fourth research challenge in this context (RC4). As shown in this study, STP approaches may interrelate to better OSH performance at the company level. The scenario calculation shown in this study shows that the costs to use STPs for a singular company are moderate. Thus speculative savings through accident prevention can be achieved with rather minimal improvements.

Concerning our rough cost-benefit analyses, we have identified four discussion points for RC4. First, cost-benefit requires a causal link between the intervention (participation in the safety training) and the effect (reduced accidents). This implies that the intervention works and is implemented correctly. Due to the dynamics of companies and $\mathrm{OSH}$ interventions, most studies report implementation problems. Hence, the arguments for claiming causality are weaker, and/or causality has a different character than RCT prescript. An analysis of the context and mechanisms in both intervention and control companies can qualify the judgment of causality. Moreover, with the inclusion of realistic evaluation, the focus is on probabilistic effects under certain circumstances (opposite of deterministic effects). Second, an OSH cost-benefit analysis should include 
a comparison of case companies where the intervention has been conducted and companies where it has not (ex-ante; perceived effects of not participating). Third, accident costs vary greatly and company and country specific insurance policies and practices should be acknowledged (Dong et al. 2007). Fourth, there can be methodological and ethical difficulties and dilemmas in measuring the exact effects of accident prevention (Rossi et al. 2004). To be valid, researchers should include the positive and negative effects of the intervention and its side effects.

\section{Conclusions}

Workplace safety research highlights the need for innovative OSH training approaches; therefore, a new STP concept is introduced. STP training involves real-life demonstrations of good and bad work environments. STP trainings are based on information sharing through different channels and following in-depth discussions by the trainer and the trainee group. The ultimate goal of STP training is to encourage and stimulate behaviour changes at individual, work group and company levels. Eventually, behaviour changes and improved OSH climate will reduce occupational accidents, injuries, and illnesses. Based on the realistic evaluation approach, evidence regarding training effectiveness is analysed on individual and on organizational levels, respectively. Our analysis shows that the trainees are greatly satisfied with STP training and report intentions to make changes in their behaviour in practice. However, the ratings of the training points vary across gender, age, and position suggesting that STP training should be tailored to each group. Results indicate that companies who use STPNF have managed to reduce their accident frequency more progressively than construction companies in Finland. A rough cost-benefit analysis of the results indicates that the STPNF not only helped reduce the number of accidents, but it also saved money. However, further studies are needed to draw a valid conclusion of the long-term 
effectiveness of STPNF training.

\section{Funding}

This work was supported by the Finnish Work Environment Fund under Grant number 114368.

\section{Disclosure statement}

No potential conflict of interest was reported by the authors.

\section{References}

Andersen, L.P., Nørdam, L, Joensson, T., Kines, P., Nielsen, K.J., 2018 Social identity, safety climate and self-reported accidents among construction workers. Construction Management and Economics, 36 (1), 22-31.

Arnold, J., Silvester, J., Patterson, F., Robertson, I., Cooper, C., Burnes, B., 2005. Work Psychology. Understanding Human Behaviour in the Workplace. 4th ed. Essex: Pearson Education Limited.

Baker, R., Brockhaus, A., Boucier, D., Chapman, L., Collins, J., Goldenhar, L., Heaney, C., Katz, T., Landsbergis, P., Martonik, J., Most, I., Schneider, S., Scharf, T., Sinclair, R., 2001. May 2000 supplement on preventing occupational injuries. American Journal of Preventive Medicine, 20 (4), 308-309.

Burgess-Limerick, R., 2018. Participatory ergonomics: Evidence and implementation lessons. Applied Ergonomics, 68 (April), 289-293.

Burke, M.J., Sarpy, S.A., Smith-Crowe, K., Chan-Serafin, S., Salvador, R.O., Islam, G., 2006. Relative effectiveness of worker safety and health training methods. American Journal of Public Health, 96 (2), 315-324.

Burke, M.J., Salvador, R., Smith-Crowe, K., Chan-Serafin, S., Smith, A.N., Sonesh, CH., 2011. The Dread Factor: How Hazards and Safety Training Influence. Journal of Applied Psychology, 96 (1), 46-70.

Confederation of Finnish Construction Industries RT. 2018. Tapaturmakyselyt ja tulokset [Occupational accident inquiries and results] [online]. Available from: http://www.rakennusteollisuus.fi/Tietoa- 
alasta/Tyoturvallisuus/Tyoturvallisuuskysely-ja-tulokset/ [Accessed 13 Mar 2018].

Cooper, L., Baber, C. 2005. Focus groups. In: N. Stanton, A., Hedge, K., Brookhuis, E., Salas, H., Hendrick, eds. Handbook of Human Factors and Ergonomics Methods. Boca Raton, FL: CRC Press, chapter 32-1.

Demirkesen, S., Arditi, D., 2015. Construction safety personnel's perceptions of safety training practices. International Journal of Project Management, 33 (5), 1160 1169.

Dong, X., Entzel, P., Men, Y., Chowdury, R., Schneider, S., 2004. Effects of safety and health training on work-related injury among construction laborers. Journal of Occupational and Environmental Medicine, 46 (12), 1222-1228.

Dong, X., Ringen, K., Yurong, M., Fujimoto, A., 2007. Medical costs and sources of payment for work-related injuries among hispanic construction workers. Journal of Occupational and Environmental Medicine, 49 (12), 1367-1375.

Drejer, I., Lund Vinding, A., 2006. Organisation, 'anchoring' of knowledge, and innovative activity in construction. Construction Management and Economics, 24 (9), 921-931.

Fisk, A.D., Rogers, W.A., Charness, N., Czaja, S.J., Sharit, J., 2009. Designing for older adults. Principles and creative human factors approaches. 2 nd ed. Boca Raton, FL: CRC Press.

Hale, A., 1984. Is safety training worthwhile? Journal of Occupational Accidents, 6 (13), 17-33.

Hendrick, H., 2003. Determining the cost-benefits of ergonomics projects and factors that lead to their success. Applied Ergonomics 34 (5), 419-427.

Hendrick, H., 2008. Applying ergonomics to systems: Some documented "lessons learned". Applied Ergonomics, 39 (4), 418-426.

Ho, C-L., Dzeng, R-J., 2010. Construction safety training via e-Learning: Learning effectiveness and user satisfaction. Computers \& Education, 55 (2), 858-867.

Hofmann, D.A., Burke, M.J., Zohar, D., 2017. 100 Years of Occupational Safety Research: From Basic Protections and Work Analysis to a Multilevel View of Workplace Safety and Risk. Journal of Applied Psychology, 102 (3), 375-388.

Holt, G.D., 2013. Construction business failure: conceptual synthesis of causal agents. Construction Innovation, 13 (1), 50-76. 
Isaacs, W.N., 1993. Taking flight: Dialogue, collective thinking, and organizational learning, Organizational Dynamics, 22 (2), 24-39.

Khosravi Y., Asilian-Mahabadi H., Hajizadeh E., Hassanzadeh-Rangi N., Bastani N., Behzadan, A.H., 2014. Factors Influencing Unsafe Behaviors and Accidents on Construction Sites: A Review, International Journal of Occupational Safety and Ergonomics, 20 (1), 111-125.

Kines, P., Andersen, L.P., Spangenberg, S., Mikkelsen, K.L., Dyreborg, J., Zohar, D., 2010. Improving construction site safety through leader-based verbal safety communication. Journal of Safety Research, 41 (5), 399-406.

Kirkpatrick, D.L., 1959. Techniques for evaluating training problems. Journal of the American Society of Training Directors, 13 (11), 3-26.

Kirschner, P.A., 2017. Stop propagating the learning style myth. Computers \& Education, 106 (March), 166-171.

Kjestveit, K., Tharaldsen, J., Holte, K.A., 2011. Young and strong: What influences injury rates within building and construction. Safety Science Monitor, 2(5), 115.

Klotz, M., 2013. Measuring the effectiveness of training in occupational health and safety. Safety Science Monitor, 2 (4), 1-16.

Komaki, J., Heinzmann, A.T., Lawson, L., 1980. Effect of training and feedback: Component analysis of a behavioral safety program. Journal of Applied Psychology, 65 (3), 261-270.

Kramer D., Bigelow, P., Carlan N., Wells, R., Garritano, E., Vi, P., Plawinski, M., 2010. Searching for needles in a haystack: Identifying innovations to prevent MSDs in the construction sector. Applied Ergonomics, 41 (4), 577-584.

Kvorning, L., Hasle, P., Christensen, U., 2015. Motivational factors influencing small construction and auto repair enterprises to participate in occupational health and safety programmes. Safety Science, 71 (January), 253-263.

Lander, F., Nielsen, K. J., Lauritsen, J., 2016. Work injury trends during the last three decades in the construction industry. Safety Science, 85 (June), 60-66.

Lehtola, M.M., Van Der Molen, F., Lappalainen, J., Hoonakker, P.L.T., Hsiao, H., Haslam, R.A., Hale, A.R., Verbeek, J.H., 2008. Review and special article: The Effectiveness of Interventions for Preventing Injuries in the Construction Industry. A Systematic Review. American Journal of Preventive Medicine, 35 (1), 77-85. 
Loosemore, M., Lee, P., 2002. Communication Problems with Ethnic Minorities in the Construction Industry. International journal of Project Management, 20 (7), $517-524$.

Manuele FA., 2011. Accident costs. Rethinking ratios of indirect to direct costs. Professional Safety, 56 (1), 39-47.

Matinaro, V., Liu, Y., 2017. Towards increased innovativeness and sustainability through organizational culture: a case study of a Finnish construction business. Journal of Cleaner Production, 142 (Part 4), 3184-3193.

Nielsen, K., Randall, R., 2013. Opening the black box: Presenting a model for evaluating organizational-level interventions. European Journal of Work and Organizational Psychology, 22 (5), 601-617.

Nielsen, K., Hansen, C.D., Bloksgaard, L., Christensen, A-D., Jensen, S.Q., Kyed, M., 2015. The impact of masculinity on safety oversights, safety priority and safety violations in two male-dominated occupations. Safety Science, 76 (June), 82-89.

Pedersen, L.M., Nielsen, K.J., Kines, P., 2012. Realistic Evaluation as a New Way to Design and Evaluate Occupational Safety Interventions. Safety Science, 50 (1), $48-54$.

Phua, F.T.T., 2017. Does the built-environment industry attract risk-taking individuals? Construction Management and Economics, 34 (4), 207-2017.

Reiman, A. Möller Pedersen, L., Väyrynen, S., Sormunen, E., Airaksinen, O., Haapasalo, H., Räsänen, T., 2019a. Safety Training Parks - Cooperative Contribution to Safety and Health Training. International Journal of Construction Education and Research, 15 (1), 19-41.

Reiman A., Airaksinen O., Fischer K., 2019b. Safety Training Parks - Cooperative Initiatives to Improve Future Workforce Safety Skills and Knowledge. In: Bagnara S., Tartaglia R., Albolino S., Alexander T., Fujita Y., eds, 20th Congress of the International Ergonomics Association, 26-30 August 2018 Florence. Springer: Cham: Advances in Intelligent Systems and Computing, vol $825,669-678$.

Reiman, A., Räisänen, T., Väyrynen, S., Autio, T., 2019c. Strategic Accident Reduction and Consequential Financial Benefits in an Energy Company. Journal of Occupational Safety and Ergonomics, 25 (1), 153-160. 
Ricci F, Chiesi A, Bisio C, Panari C, Pelosi A., 2016. Effectiveness of occupational health and safety training: A systematic review with meta-analysis. Journal of workplace learning, 28 (6), 355-377.

Rikhardsson PM., 2004. Accounting for the cost of occupational accidents. Corporate Social Responsibility and Environmental Management, 11(2), 63-70.

Ringen, K., van Duivenbooden, J.C., Melius, J., 2010. Construction safety and health Foreword. American Journal of Industrial Medicine, 53 (6), 551.

Robson, L.S., Stephenson, C.M., Schulte, P.A., Amick, B.A. III, Irvin, E.L., Eggerth, D.E., Chan, S., Bielecky, A.R., Wang, A.M., Heidotting, T.L., Peters, R.H., Clarke, J.A., Cullen, K., Rotunda, C.J., Grupp, P.L., 2012. A systematic review of the effectiveness of occupational health and safety training. Scandinavian Journal of Work, Environment \& Health, 38 (3), 193-208.

Rossi, P.H., Lipsey, M.W., Freeman, H.E., 2004. Evaluation. A Systematic Approach. 7th ed. London: Sage Publications Inc.

Sacks, R., Perlman, A., Barak, R., 2013. Construction safety training using immersive virtual reality. Construction Management and Economics, 31 (9), 1005-1017.

Salminen, S., 2004. Have young workers more injuries than older ones? An international literature review. Journal of Safety Research, 35 (5), 513-521.

Sanson-Fisher, R.W., Bonevski, B., Green, L.W., D'Este, C., 2007. Limitations of the Randomized Controlled Trial in Evaluating Population-Based Health Interventions. American Journal of Preventive Medicine, 33 (2), 155-161.

Seppänen, T., 2009. Absence from work - Finland. Observatory EUR WORK 2010 [online]. European Observatory of Working Life (EU). Available from: http://www.eurofound.europa.eu/observatories/eurwork/comparativeinformation/nationalcontributions/finland/absence-from-work-finland. [Accessed 5 May 2019].

Swuste, P., Frijters, A., Guldenmund, F.W., 2012. Is it possible to influence safety in the building sector? A literature review extending from 1980 until the present. Safety Science, 50 (5), 1333-1343.

Ulubeyli, S., Arslan, V., Kivrak, S., 2015. A semiotic analysis of cartoons about occupational health and safety issues in the construction workplace. Construction Management and Economics, 33 (5-6), 467-483.

Verbeek, J.H., Martimo, K.P., Karppinen, J., Kuijer, P.P.F.M., Viikari-Juntura, E., Takala, E.P., 2011. Manual material handling advice and assistive devices for 
preventing and treating back pain in workers. Cochrane Database of Systematic Reviews, 15 (6), CD005958.

Wilkins, J.R., 2011. Construction workers' perceptions of health and safety training programmes. Construction Management and Economics, 29 (10), 1017-1026.

Yassi, A., Lockhart, K., Sykes, M., Buck, B., Stime, B., Spiegel, J. M., 2013. Effectiveness of Joint Health and Safety Committees: A Realist Review. American Journal of Industrial Medicine, 56 (4), 424-438.

Yrjämä-Huikuri L, Väyrynen S., 2015. The total risk of lost time accidents for personnel of two large employers in Finland. In: Väyrynen S, Häkkinen K, Niskanen T, eds. Integrated occupational safety and health management. Solutions and industrial cases. Cham: Springer, 129-143.

Zhao, D., Lucas, J., 2015. Virtual reality simulation for construction safety promotion. International Journal of Injury Control and Safety Promotion, 22 (1), 57-67.

Zohar, D., Luria, G., 2005. A Multilevel Model of Safety Climate: Cross-Level Relationships between Organization and Group-Level Climates. Journal of Applied Psychology, 90 (4), 616-628.

Zohar, D., 2010. Thirty years of safety climate research: Reflections and future directions. Accident Analysis \& Prevention, 42 (5), 1517-1522.

Zou, P.X.W., Sunindijo, R.Y., 2015. Strategic Safety Management in Construction and Engineering. 3rd ed. Chichester, UK: Wiley-Blackwell. 